\title{
WEIGHT CENTRALIZER EXPECTATIONS WITH FINITE INDEX
}

\author{
ESTEBAN ANDRUCHOW* and ALEJANDRO VARELA
}

\begin{abstract}
Let $M$ be a von Neumann algebra, $\varphi$ be a faithful, normal semifinite weight on $M$ and $M^{\varphi}$ its centralizer. We characterize the conditional expectations $E_{\varphi}: M \rightarrow M^{\varphi}$ of finite index for a faithful normal strictly semifinite weight $\varphi$ on a semifinite von Neumann algebra $M$ with finite dimensional center. This result is used to characterize weights $\varphi$ such that the orbit $U_{\varphi}=\{\varphi \circ \operatorname{Ad}(u): u$ unitary in $M\}$ can be represented as a submanifold of $M_{1}$ (=basic extension of $\left.E_{\varphi}: M \rightarrow M_{\varphi}\right)$.
\end{abstract}

1.1 Introduction. Let $M$ be a von Neumann algebra and $\varphi$ a faithful, normal and semifinite weight on $M$. Denote by $\sigma_{t}^{\varphi}$ the modular group of $\varphi$ and by $M^{\varphi}$ the centralizer of $\varphi$, i.e. the subalgebra of $M$ of fixed points for $\sigma_{t}^{\varphi}$. It is well known ([C],[T]) that there exists a faithful and normal conditional expectation $E_{\varphi}: M \rightarrow M^{\varphi}$ satisfying $\varphi \circ E_{\varphi}=\varphi$ if and only if the weight $\varphi$ is semifinite in $M^{\varphi}$. Under these asumptions, the expectation $E_{\varphi}$ is unique. Such weights $\varphi$ are called strictly semifinite [C].

There are two notions of finite index for a conditional expectation between von Neumann algebras [BDH], [Ha]. In many cases they do coincide (for example, if the algebras are factors). In general they do not coincide ([FK]). In this paper we investigate the finiteness (in the two senses) of the expectations $E_{\varphi}$ associated with weights $\varphi$. Very naturally one is constrained to consider semifinite algebras. In section 4 we state that in the case of factors (or more generally algebras with finite dimensional center) the finite index condition holds if and only if the spectrum of the Radon-Nykodim derivative of $\varphi$ with respect to a tracial weight (see [PT]) has finite spectrum.

Denote by $\mathscr{U}(M)$ the unitary group of $M$. Our interest in the inclusions $M^{\varphi} \subset M$ is motivated by the geometric study of the orbits

$$
\mathscr{U}_{\varphi}=\{\varphi \circ \operatorname{Ad}(u): u \in \mathscr{U}(M)\} .
$$

In 5.1 we show that for the case $M=\mathscr{L}(H)$ the unitary orbit of a faithful

\footnotetext{
* Supported by UBACYT (Argentina).

Received February 10, 1997.
} 
and normal state is never a topological submanifold of $\mathscr{L}(H)^{*}$. Moreover, in the case of infinite weights, the orbit can not even be regarded as functionals on a suitable Banach space.

On the other hand, using the appropriate quotient topology, this sets can be regarded as homogeneous spaces (i.e. quotients of Banach-Lie groups with a differentiable structure). Namely, $\mathscr{U}_{\varphi} \simeq \mathscr{U}(M) / \mathscr{U}\left(M^{\varphi}\right)$.

A reductive structure for a homogeneous space $G / K$ is an invariant supplement for the Lie-Banach algebra $\mathscr{K}$ of $K$ in the Lie-Banach algebra $\mathscr{G}$ of $G$, where invariant means invariant under the natural action of $K$ in $\mathscr{G}$.

In our case, such a supplement is given by $\operatorname{Ker}\left(E_{\varphi}\right)$. Moreover, by means of the expectation $E_{\varphi}$ one may obtain a representation of $\mathscr{U}_{\varphi}$ as a space of projections in a von Neumann superalgebra $M_{1}$ of $M$. Recall the basic construction associated to a ( $\varphi$ invariant) conditional expectation. This yields a projection $e_{\varphi}$ (denoted the Jones projection of $E_{\varphi}$ ) acting on the Hilbert space $H_{\varphi}$ of the GNS triple of $\varphi$. As is usual notation, $M_{1}$ is the von Neumann algebra generated by $e_{\varphi}$ and $M$. Then $e_{\varphi}$ satisfies

i) $e_{\varphi} m e_{\varphi}=E_{\varphi}(m) e_{\varphi}, m \in M$

ii) $\left\{e_{\varphi}\right\}^{\prime} \cap M=M^{\varphi}$

iii) $e_{\varphi} M_{1} e_{\varphi}$ is isomorphic to $M^{\varphi}$ via the ${ }^{*}$-isomorphism $x \mapsto x e_{\varphi}=e_{\varphi} x e_{\varphi}$. Then, as in [AS2], one has the (one to one) representation

$$
\begin{gathered}
\mathscr{U}_{\varphi} \hookrightarrow\left\{\text { projections of } M_{1}\right\} \\
\varphi \circ \operatorname{Ad}(u) \mapsto u^{*} e_{\varphi} u
\end{gathered}
$$

which is continuous if $\mathscr{U}_{\varphi}$ is considered with the quotient topology $\mathscr{U}(M) / \mathscr{U}\left(M^{\varphi}\right)$ and the set of projections with the norm topology of $M_{1}$.

If the index of $E_{\varphi}$ is finite, then this map is a topological imbedding (i.e. a homeomorphism between $\mathscr{U}_{\varphi}$ and $\left.\mathscr{U}_{M}\left(e_{\varphi}\right)=\left\{u^{*} e_{\varphi} u: u \in \mathscr{U}(M)\right\}\right)$. Moreover, in [AS] it was shown that $\mathscr{U}_{M}\left(e_{\varphi}\right)$ is a $\mathrm{C}^{\infty}$ submanifold of $M_{1}$ if and only if the index of $E_{\varphi}$ is finite. Therefore, the finite index condition allows one to regard $\mathscr{U}_{\varphi}$ as a $\mathrm{C}^{\infty}$ submanifold of the space of projections of $M_{1}$ (which is a space with rich geometric structure [CPR] and itself a submanifold of $M_{1}$ ). If the index is infinite, one still has the continuous representation (called basic representation) $\mathscr{U}_{\varphi} \hookrightarrow$ projections of $\left.M_{1}\right\}$.

If the index of $E_{\varphi}$ is infinite, the above representation is a homeomorphism if one changes the usual norm of $M$ by the $M^{\varphi}$-Hilbert module norm induced by $E_{\varphi}$. The price payed is that $M$ is not complete with this new norm (completeness being equivalent to the finite index condition). Section 5 is devoted to the description of this application.

1.2 Preliminary and notations. Throughout this paper $\varphi$ will denote a faithful normal semifinite weight and $\sigma_{t}^{\varphi}$ the modular group of $\varphi$. Denote by 
$M^{\varphi}=\left\{x \in M: \sigma_{t}^{\varphi}(x)=x, \forall t \in \mathrm{R}\right\}$. A strictly semifinite weight is a semifinite weight of $M$ such that $\left.\varphi\right|_{M^{\varphi}}$ is also semifinite on $M^{\varphi}$.

As it is standard notation, $\mathscr{N}_{\varphi}=\left\{x \in M: \varphi\left(x^{*} x\right)<\infty\right\}$ and $\left(H_{\varphi}, \pi_{\varphi}, \eta_{\varphi}\right)$ denotes the GNS triple for $\varphi$, i.e. $H_{\varphi}$ is the completion of $\mathscr{N}_{\varphi}$ to a Hilbert space, $\pi_{\varphi}: M \longrightarrow \mathscr{L}\left(H_{\varphi}\right)$ is the usual $*$-isomorphism, $\eta_{\varphi}: \mathscr{N}_{\varphi} \longrightarrow H_{\varphi}$ is the canonical imbedding, with

$$
\left\langle\eta_{\varphi}(y), \eta_{\varphi}(z)\right\rangle=\varphi\left(z^{*} y\right) \text { and } \pi_{\varphi}(z) \eta_{\varphi}(y)=\eta_{\varphi}(z y) \text { for } y, z \in \mathscr{N}_{\varphi}, z \in M
$$

If $G$ is a locally compact group, an invariant mean $m$ on $G$ is a state of $L^{\infty}(G)$ such that $m$ is invariant under the action of $G$, i.e. $m\left(f\left(t_{0}.\right)\right)=m(f()$. for all $t_{0} \in G$ and $f \in L^{\infty}(G)$ (see [Pa]).

Let $m$ be an invariant mean in $\mathrm{R}$, and $\alpha: \mathbf{R} \longrightarrow \operatorname{Aut}(M)\left(\alpha(t)=\alpha_{t},\right)$ a weakly continuous homomorphism of $\mathrm{R}$ on the automorphism group of a von Neumann algebra $M$ represented on $\mathscr{L}(H)$. For $x \in M$ denote by $\int_{\mathrm{R}} \alpha_{t}(x) d m(t)$ the element of $M$ given by

$$
\left\langle\left(\int_{\mathrm{R}} \alpha_{t}(x) d m(t)\right) \xi, \eta\right\rangle=m\left(t \mapsto<\alpha_{t}(x) \xi, \eta>\right), \text { for } \xi, \eta \in H
$$

This integral does not depend on the Hilbert space $H$.

Let $E: M \rightarrow N$ be a normal conditional expectation and let $\psi$ be a normal state of $N$. Denote also by $\psi$ the extension of this state to $M$ given by $\psi \circ E$. Let $e$ be the Jones projection of $E$ (and $\psi$ ), i.e. the orthogonal projection obtained as the closure of $E$ as an operator on $L^{2}(M, \psi)$ (with range $L^{2}(N, \psi)$ ). As it is also standard, denote by $M_{1}$ the algebra generated by $M$ and $e$ in $\mathscr{L}\left(L^{2}(M, \psi)\right)$.

After the introduction of Jones paper [Jo] several notions of index for a conditional expectation between von Neumann algebras appeared ([PP], $[\mathrm{K}],[\mathrm{W}])$. We will follow the terminology of the paper by Baillet, Denizeau and Havet [BDH], where three notions of index are considered. Let $N \subset M$ be a von Neumann subalgebra and $E: M \rightarrow N$ a conditional expectation.

$E$ is said to be of weak finite index if there exists a positive real number $\lambda$ such that $E-\lambda \mathrm{Id}$ is a positive map. In that case put $\operatorname{Ind}_{w}(E):=\lambda_{0}^{-1}$ where $\lambda_{0}$ is the supremum of all such $\lambda$ 's (the index is said to be $\infty$ if no such $\lambda$ exists).

In such case ([Po], [BDH], [FK]), there exists another positive constant $\kappa$ such that $E-\kappa \mathrm{Id}$ is completely positive, and there is a family $\left\{m_{i}\right\}_{i \in I} \subset M$ such that

i) $1=\sum_{i \in I} m_{i} e m_{i}^{*}$, ( $e$ the Jones projection associated to $E$ )

ii) $E\left(m_{i}^{*} m_{j}\right)=\delta_{i j} p_{i},\left(p_{i}\right.$ projections in $\left.N\right)$

iii) and $\sum_{i \in I} m_{i} m_{i}^{*}$ converges ultraweakly in $M$. 
In $[\mathrm{BDH}]$ it is shown that the limit of the latter sum belongs to the center of $M$, and is called the index of $E$, and will be denoted by $\operatorname{Ind}(E)$.

It is said that $E$ has strongly finite index if the family $\left\{m_{i}\right\}_{i \in I}$ is finite.

There are conditional expectations that are of finite index and not of strongly finite index $[\mathrm{J}],[\mathrm{FK}]$. Nevertheless these two notions of finite index conincide in many cases, for instance if $N$ is a subfactor [BDH].

\section{Conditional expectations and invariant means}

REMARK 2.1. Let $m$ be an invariant mean in $\mathrm{R}, \varphi$ a faithful normal semifinite weight on a von Neumann algebra $M$, and $E_{\varphi}^{m}: M \longrightarrow M$ the map

$$
E_{\varphi}^{m}(x)=\int_{\mathrm{R}} \sigma_{t}^{\varphi}(x) d m(t)
$$

Then $E_{\varphi}^{m}$ is a $\sigma_{t}^{\varphi}$-invariant conditional expectation with range $M^{\varphi}$ which satisfies

$$
\varphi\left(E_{\varphi}^{m}(x)\right) \leq \varphi(x) \quad \text { for all } x \in M_{+} .
$$

Moreover, if $\varphi$ is strictly semifinite, then $E_{\varphi}^{m}$ is the unique faithful and normal conditional expectation invariant with $\varphi$.

Proof. The fact that $E_{\varphi}^{m}$ is a $\sigma^{\varphi}$ invariant conditional expectation is well known (see for instance $10.12[\mathrm{~S}]$ ).

In order to verify that $\varphi\left(E_{\varphi}^{m}(x)\right) \leq \varphi(x)$ for every $x>0$, suppose $M$ represented in a standard form. Therefore $\varphi(z)=\sup _{i}\left\langle z \xi_{i}, \xi_{i}\right\rangle$ for certain vectors $\xi_{i}, i \in I$. Then

$$
\varphi\left(E_{\varphi}^{m}(x)\right)=\sup _{i}\left\langle E_{\varphi}^{m}(x) \xi_{i}, \xi_{i}\right\rangle=\sup _{i} m\left(t \mapsto\left\langle\sigma_{t}^{\varphi}(x) \xi_{i}, \xi_{i}\right\rangle\right) \leq \varphi(x)
$$

where the last inequality holds because $\left\langle\sigma_{t}^{\varphi}(x) \xi_{i}, \xi_{i}\right\rangle \leq \varphi\left(\sigma_{t}^{\varphi}(x)\right)=\varphi(x)$.

Let us suppose now that $\varphi$ is strictly semifinite. We will see that under this hypothesis $E_{\varphi}^{m}$ is faithful, normal and $\varphi \circ E_{\varphi}^{m}=\varphi$, and therefore coincides with Takesaki's expectation.

Proof. Let $x \in M_{+}$. Since $\varphi$ is strictly semifinite, there exists a normal positive linear functional $\psi$ which is $\sigma_{t}^{\varphi}$ invariant such that $\psi(x)>0$. Then we can write $\psi=\varphi\left(h\right.$.) whith $h$ a positive operator affiliated to $Z\left(M^{\varphi}\right)$, and for certain $h_{\epsilon} \in Z\left(M^{\varphi}\right)$ (see [P]),

$$
\psi\left(E_{\varphi}^{m}(x)\right)=\varphi\left(h E_{\varphi}^{m}(x)\right)=\lim _{\epsilon \longrightarrow 0} \varphi\left(h_{\epsilon}^{1 / 2} E_{\varphi}^{m}(x) h_{\epsilon}^{1 / 2}\right) .
$$

Note that $\varphi\left(h_{\epsilon}\right)<\infty$ (i.e. $h_{\epsilon}^{1 / 2} \in \mathscr{N}_{\varphi}$ ) and 


$$
\begin{aligned}
\varphi\left(h_{\epsilon}^{1 / 2} E_{\varphi}^{m}(x) h_{\epsilon}^{1 / 2}\right) & =\left\langle E_{\varphi}^{m}(x) \eta_{\varphi}\left(h_{\epsilon}^{1 / 2}\right), \eta_{\varphi}\left(h_{\epsilon}^{1 / 2}\right)\right\rangle \\
& =m\left(t \mapsto\left\langle\sigma_{t}^{\varphi}(x) \eta_{\varphi}\left(h_{\epsilon}^{1 / 2}\right), \eta_{\varphi}\left(h_{\epsilon}^{1 / 2}\right)\right\rangle\right) \\
& =m\left(t \mapsto \varphi\left(h_{\epsilon}^{1 / 2} \sigma_{t}^{\varphi}(x) h_{\epsilon}^{1 / 2}\right)\right)=m\left(t \mapsto \varphi\left(\sigma_{t}^{\varphi}\left(h_{\epsilon}^{1 / 2} x h_{\epsilon}^{1 / 2}\right)\right)\right) \\
& =\varphi\left(h_{\epsilon}^{1 / 2} x h_{\epsilon}^{1 / 2}\right) .
\end{aligned}
$$

Therefore $\psi\left(E_{\varphi}\right)=\psi(x)>0$, which proves that $E_{\varphi} m(x) \neq 0$ and then $E_{\varphi}^{m}$ is faithful.

Let us show that $E_{\varphi}^{m}$ is normal. If $p_{i}, p \in M$, and $p_{i} \nearrow p$, then $E_{\varphi}^{m}\left(p_{i}\right) \nearrow i \rightarrow \sup E_{\varphi}^{m}\left(p_{i}\right)=q \in M^{\varphi}$. Clearly $q \leq E_{\varphi}^{m}(p)$. Suppose $E_{\varphi}^{m}(p)-q \neq 0$. Since $\varphi$ is strictly semifinite, there exists a normal positive linear functional $\psi$ which is $\sigma_{t}^{\varphi}$ invariant such that $\psi\left(E_{\varphi}^{m}(p)-q\right)>0$. As before, this $\psi$ verifies $\psi\left(E_{\varphi}^{m}(y)\right)=\psi(y)$ for $y \in M$, and then

$$
\begin{aligned}
\psi\left(E_{\varphi}^{m}(p)-q\right) & =\psi\left(E_{\varphi}^{m}(p)-E_{\varphi}^{m}(q)\right)=\psi(p-q) \\
& =\sup _{i} \psi\left(p_{i}\right)-\sup _{i} \psi\left(E_{\varphi}^{m}\left(p_{i}\right)\right)=0
\end{aligned}
$$

Therefore $E_{\varphi}^{m}\left(\sup _{i} p_{i}\right)=E_{\varphi}^{m}(p)$ which proves the normality of $E_{\varphi}^{m}$.

It remains to verify that $\varphi \circ E_{\varphi}^{m}=\varphi$. Since $\varphi$ is strictly semifinite there exist normal positive functionals $\psi_{i}$ with orthogonal supports $p_{i}$ such that $\varphi=\sum_{i} \psi_{i}$. Therefore, $\varphi\left(p_{i}.\right)=\psi_{i}$ with $p_{i} \in M^{\varphi}$, and then $\psi_{i} \circ \sigma_{t}^{\varphi}=\psi_{i}$. If we represent $M$ in a standard form, there exist $\xi_{i}$ such that $\psi_{i}(x)=\left\langle x \xi_{i}, \xi_{i}\right\rangle$ for all $x \in M$, and then

$\psi_{i}\left(E_{\varphi}^{m}(x)\right)=\left\langle E_{\varphi}^{m}(x) \xi_{i}, \xi_{i}\right\rangle=m\left(t \mapsto\left\langle\sigma_{t}^{\varphi}(x) \xi_{i}, \xi_{i}\right\rangle\right)=m\left(t \mapsto \psi_{i}\left(\sigma_{t}^{\varphi}(x)\right)\right)=\psi_{i}(x)$.

Therefore, $\varphi\left(E_{\varphi}^{m}(x)\right)=\sum_{i} \psi_{i}\left(E_{\varphi}^{m}(x)\right)=\sum_{i} \psi_{i}(x)=\varphi(x)$, which completes the proof.

In other words, if $\varphi$ is a faithful normal strictly semifinite weight then $E_{\varphi}^{m}$ does not depend on the choice of $m$.

Remark 2.2. If $J \subset \mathrm{R}$ is an interval, put $\Psi_{J}(x)=|J|^{-1} \int_{J} x(t) d t$. This positive forms are called Bohr means. Eberlein ([E] Th. 5.2) showed that if $x$ is almost periodic then the net $\left\{\Psi_{J}(x)\right\}_{J \subset R}$ converges to a unique limit. Using this result, applied to $x(t)=\left\langle\sigma_{t}^{\varphi}(x) \xi, \eta\right\rangle$, it can be proven directly for almost periodic weights (i.e. weights $\varphi$ such that the modular operator $\Delta_{\varphi}$ is diagonalizable) that $\left\langle E_{\Psi_{J}}(x) \xi, \eta\right\rangle$ converges to a unique limit, namely $E_{\varphi}(x)$. Note that almost periodic weights are strictly semifinite.

Example 2.3. If the weight $\varphi$ of 2.1 fails to be strictly semifinite, the expectation $E_{\varphi}^{m}$ may be neither normal nor faithful, and depends on the choice of $m$. Let $m$ be a mean for R obtained as in 2.2. Let $M=\mathscr{L}\left(L^{2}(0,1)\right), h=\mu_{x}$ 
the operator "multiplication by $x$ " and $\varphi(x)=\operatorname{Tr}(h x)$ where $\operatorname{Tr}$ is the trace of $M$. It is straightforward to verify that $M^{\varphi}=\{h\}^{\prime}=L^{\infty}(0,1)$ regarded as multiplication operators $\mu_{f}$ in $L^{2}(0,1)$. Therefore in this case, for all $a \in M$

$$
E_{\varphi}^{m}(a)=\int_{\mathrm{R}} \mu_{x^{i t}} a \mu_{x^{-i t}} d m(t) .
$$

We will show that these expectations coincide with limit points of the so called von Neumann's operation [U], i.e. expectations obtained as cluster points of the net of expectations

$$
\left\{E_{\Pi}(a)=\sum_{\Delta \in \Pi} \mu_{\chi_{\Delta}} a \mu_{\chi_{\Delta}}: \Pi \text { finite partitions of }(0,1)\right\}
$$

where $\chi_{\Delta}$ is the characteristic function of $\Delta \in \Pi$, the partitions are directed by inclusion and the topology is that of weak operator convergence at every $a \in M$. In [Su] it was shown that there exists an uncountable set of limit points for this net. Moreover, this limit points are conditional expectations which have the compact operators in their kernels, and therefore can not be normal nor faithful.

Let $\sum_{i} \alpha_{i} \chi_{J_{i}}$ be a step function close to the identity function $t$ in the norm topology of $L^{\infty}(0,1)$. Then for every $a \in \mathscr{L}\left(L^{2}(0,1)\right)$ we have that $E_{\varphi}^{m}(a)$ is close to

$$
\begin{gathered}
\int_{\mathrm{R}} \sum_{n, m} \alpha_{n}^{i t} \alpha_{m}^{-i t} \mu_{\chi_{J_{n}}} a \mu_{\chi_{J_{m}}} d m(t)= \\
=\sum_{n \neq m} \mu_{\chi_{J_{n}}} a \mu_{\chi_{J_{m}}} \int_{\mathrm{R}} \alpha_{n}^{i t} \alpha_{m}-i t d m(t)+\sum_{n} \mu_{\chi_{J_{n}}} a \mu_{\chi_{J_{n}}} .
\end{gathered}
$$

It is easy to verify that for $n \neq m$ (with invariant means obtained as weak limits of Bohr means) $\int_{\mathrm{R}} \alpha_{n}^{i t} \alpha_{m}^{-i t} d m(t)=0$. Therefore $E_{\varphi}^{m}(a)$ can be approximated (in the weak operator topology) by the sums

$$
\sum_{n} \mu_{\chi_{J_{n}}} a \mu_{\chi_{J_{n}}}=E_{I I}(a)
$$

if $\Pi$ is the partition given by $J_{1}, \ldots, J_{n}$.

Suarez showed in [Su] that the expectations obtained as limits of the $E_{\Pi}$ when restricted to the operators which are diagonal in the Fourier basis take scalar values. Denote by $\left\{e_{n}\right\}_{n \in Z}$ the Fourier basis of $L^{2}(0,1)$. Then for each invariant mean $m$ of $\mathrm{R}$ the linear functional

$$
l^{\infty}(\mathbf{Z}) \ni\left\{\lambda_{n}\right\} \mapsto E_{\varphi}^{m}\left(\sum_{n} \lambda_{n} e_{n} \otimes e_{n}\right) \in \mathrm{C}
$$


is an invariant mean for Z. Moreover, any mean in Z may be obtained in this fashion $[\mathrm{Su}]$.

\section{Conditional expectations with finite index}

Kosaki proved (see [W], Prop. 2.5.2) that if $M \supset N$ are factors and $E: M \longrightarrow N$ is a faithful and normal conditional expectation of finite index, then $M$ is finite iff $N$ is finite. Jolissaint [J] generalized this result for non factors (with the finite index condition). Let us state this result.

Proposition 3.1. Let $M$ be a von Neumann algebra and $E: M \longrightarrow N a$ faithful and normal conditional expectation.

a) If $N$ is finite and $E$ is of strongly finite index then $M_{1}$ and $M$ are finite.

b) If $N$ is semifinite and $E$ is of finite index then $M_{1}$ and $M$ are semifinite.

Proof. a): Let $\varphi$ be a faithful normal state on $N$, then $\varphi \circ E$ is a faithful normal state on $M$. Let us also denote by $\varphi$ this extension. Let $\left(H_{\varphi}, \pi_{\varphi}, \xi_{\varphi}\right)$ be the GNS triple for $\varphi$ and $M$ and $J_{\varphi}=J$. Since $N$ is finite, $N^{\prime}$ is semifinite. Therefore, $M_{1}=J N^{\prime} J$ is also semifinite.

The projection $\left[N^{\prime} \xi_{\varphi}\right] \in N$ is finite. Recall ([KR] 9.1.2) that $\left[N^{\prime} \xi_{\varphi}\right] \in N$ is finite iff $\left[N \xi_{\varphi}\right] \in N^{\prime}$ finite. Therefore $\left[N \xi_{\varphi}\right]=[N .1]=e \in N^{\prime}$ is finite, and then $J e J=e$ is a finite projection in $M_{1}$. Then there exists a semifinite faithful tracial weight $\tau$ on $c(e) M_{1}=M_{1}$ such that $\tau(e)<\infty$ ([P], 5.4.6).

Since $E: M \longrightarrow M^{\varphi}$ is of strongly finite index, $1=\sum_{i=1}^{k} m_{i} e m_{i}^{*}$ for certain $m_{i} \in M$. Then

$$
\tau\left(m_{i} e m_{i}^{*}\right)=\tau\left(m_{i} e\left(m_{i} e^{*}\right)\right)=\tau\left(e m_{i}^{*} m_{i} e\right) \leq \tau\left(\left\|m_{i}\right\|^{2} e\right)<\infty .
$$

This shows that $\tau(1)<\infty$, and therefore $\tau$ is a finite trace and $M_{1}$ and $M$ are finite.

b): As in the previous point, $M_{1}$ is semifinite. Since the index of $E$ is finite, by $[\mathrm{BDH}] 3.10$ there exists a faithful and normal conditional expectation $E_{1}: M_{1} \rightarrow M$. Then it follows that $M$ is semifinite.

COROLlaRY 3.2. Let $\varphi$ be a faithful normal strictly semifinite weight in $M$.

a) If $\varphi$ is finite and $E_{\varphi}: M \longrightarrow M^{\varphi}$ has strongly finite index then $M$ is finite.

b) If $E_{\varphi}: M \longrightarrow M^{\varphi}$ has finite index then $M$ is semifinite.

\section{Characterization of $E_{\varphi}$ with finite index}

In view of 3.2 , if one will look for $E_{\varphi}$ with finite index one has to consider semifinite algebras.

Lemma 4.1. Let $M$ be a semifinite von Neumann algebra, $\tau$ a tracial weight 
on $M, \varphi=\tau\left(h\right.$.) a faithful normal strictly semifinite weight on $M$. If $\left\{p_{i}\right\}_{i \in I}$ are orthogonal spectral projections of $h$ with $\sum_{i \in I} p_{i}=1$, then

$$
E_{\varphi}(x)=E_{\varphi}\left(\sum_{i \in I} p_{i} x p_{i}\right)
$$

If moreover $\sigma(h)=\left\{r_{i}: i \in \mathbf{N}\right\}$ is discrete, then

$$
E_{\varphi}(x)=\sum_{i \in \mathrm{N}} p_{i} x p_{i}
$$

with $p_{i}$ the spectral projection of $h$ associated to $\left\{r_{i}\right\}$.

Proof. Note that $p_{i} \in Z\left(M^{\varphi}\right)$ and therefore

$$
E_{\varphi}\left(\sum_{i} p_{i} x p_{i}\right)=\sum_{i \in \mathrm{N}} E_{\varphi}\left(p_{i} x p_{i}\right)=\sum_{i \in \mathrm{N}} p_{i} E_{\varphi}(x) p_{i}=\left(\sum_{i \in \mathrm{N}} p_{i}\right) E_{\varphi}(x)=E_{\varphi}(x) .
$$

Suppose now that $\sigma(h)=\left\{r_{i}: i \in \mathrm{N}\right\}$, then $h=\sum_{i} r_{i} p_{i}$. Put $E(x)=$ $\sum_{i} p_{i} x p_{i} . \quad E$ is a faithful and normal conditional expectation onto $\left\{p_{i}: i \in \mathbf{N}\right\}^{\prime} \cap M=\{h\}^{\prime} \cap M=M^{\varphi}$. Note that it is also $\varphi$ invariant:

$$
\begin{aligned}
\varphi(E(x)) & =\varphi\left(\sum_{i} p_{i} x p_{i}\right)=\sum_{i} \tau\left(h p_{i} x p_{i}\right)=\sum_{i} \tau\left(r_{i} p_{i} x\right)=\tau\left(\sum_{i} r_{i} p_{i} x\right) \\
& =\varphi(x) .
\end{aligned}
$$

Therefore $E=E_{\varphi}$.

THEOREM 4.2. Let $M$ be a finite von Neumann algebra with finite dimensional center, $\varphi$ a faithful normal strictly semifinite weight on $M$ and $E_{\varphi}: M \longrightarrow M^{\varphi}$ the unique normal and faithful conditional expectation that leaves $\varphi$ invariant. Let $\tau$ be a faithful and normal trace in $M$ and $h$ affiliated to $Z\left(M^{\varphi}\right)$ such that $\varphi=\tau(h$.$) . The following statements are equivalent:$

(i) $E_{\varphi}$ has strongly finite index.

(ii) $E_{\varphi}$ has weakly finite index.

(iii) $\sigma(h)$ is finite.

And in particular, $\varphi$ is finite.

Proof. As $\operatorname{dim}(Z(M))<\infty$, we can suppose $M=\oplus_{1 \leq i \leq n} M_{i}$, with $M_{i}$ a finite factor. Then $\left.E_{\varphi}\right|_{M_{i}}: M_{i} \longrightarrow M_{i}^{\varphi}$ defines a conditional expectation (because $\left.Z(M) \subset M^{\varphi}\right), E_{\varphi}=\left.\sum_{i=1}^{n} E_{\varphi}\right|_{M_{i}}$, and $E_{\varphi}$ has strongly finite index iff each $\left.E_{\varphi}\right|_{M_{i}}$ does. Moreover, if $h$ is affiliated to $Z\left(M^{\varphi}\right)$, then $h=\sum_{i=1}^{n} h_{i}$ with $h_{i}$ affiliated to $Z\left(M_{i}^{\varphi}\right)$, and the spectrum of $h$ is finite iff the spectrum of each $h_{i}$ is finite. Therefore, we can suppose that $M$ is a finite factor.

(i) $\Longrightarrow$ (ii) is trivial. 
(iii) $\Longrightarrow$ (i): If $\sigma(h)=\left\{r_{1}\right\}$, then $h=r_{1} 1$. In this case $\varphi=r_{1} \tau$, and therefore $E_{\varphi}=I d$ which has strongly finite index.

Reasoning by induction, we are going to asume that the proposition is valid for $\sigma(h)=\left\{r_{i}: 1 \leq i \leq n\right\}$ and prove it for the case $n+1$. Then, if $\left\{p_{i}\right\}_{1 \leq i \leq n}$ are the orthogonal spectral projections of $h$ corresponding to $\left\{r_{i}\right\}$ with $1 \leq i \leq n$, there can be found finite $E_{\varphi}$-orthonormal $m_{k} \in M, 1 \leq k \leq z$, such that for all $x \in M, x=\sum_{k=1}^{z} m_{k} E_{\varphi}\left(m_{k}^{*} x\right)$. The existence of such family is equivalent to the strongly finite index condition of $E_{\varphi}$ (see [BDH]).

Suppose that $\sigma(h)=\left\{r_{i}: 1 \leq i \leq n+1\right\}$, and $\left\{p_{i}\right\}_{1 \leq i \leq n+1}$ are the orthogonal spectral projections of $h$ corresponding to $\left\{r_{i}\right\}_{1 \leq i \leq n+1} \subset \sigma(h)$. As $M$ is a factor, we can suppose $p_{i} \preceq p_{i+1}$ for $1 \leq i \leq n$.

We can assume the theorem is valid for the factor $\left(1-p_{n+1}\right) M\left(1-p_{n+1}\right)$, the expectation $\left.E_{\varphi}\right|_{\left(1-p_{n+1}\right) M\left(1-p_{n+1}\right)}$ and the operator $h_{n}=\sum_{i=1}^{n} r_{i} p_{i}$. Since $h$ is affiliated to $Z\left(M^{\varphi}\right)$, then $p_{i} \in Z\left(M^{\varphi}\right)$, for $1 \leq i \leq n+1$, and then

$$
\begin{aligned}
E_{\varphi}\left(\left(1-p_{n+1}\right) x\left(1-p_{n+1}\right)\right) & =E_{\varphi}\left(\left(\sum_{i=1}^{n} p_{i}\right) x\left(\sum_{i=1}^{n} p_{i}\right)\right) \\
& =\left(\sum_{i=1}^{n} p_{i}\right) E_{\varphi}(x) \sum_{i=1}^{n} p_{i} \\
& =\left(1-p_{n+1}\right) E_{\varphi}(x)\left(1-p_{n+1}\right)=\left(1-p_{n+1}\right) E_{\varphi}(x) .
\end{aligned}
$$

Therefore $E_{\varphi}\left(\left(1-p_{n+1}\right) M\left(1-p_{n+1}\right)\right) \subset\left(1-p_{n+1}\right) M\left(1-p_{n+1}\right)$. There exist orthonormal $m_{i} \in\left(1-p_{n+1}\right) M\left(1-p_{n+1}\right)$ with $1 \leq i \leq z$, such that

$$
\begin{aligned}
\left(1-p_{n+1}\right) x\left(1-p_{n+1}\right) & =\sum_{i=1}^{z} m_{i} E_{\varphi}\left(m_{i}^{*}\left(1-p_{n+1}\right) x\left(1-p_{n+1}\right)\right) \\
& =\sum_{i=1}^{z} m_{i}\left(1-p_{n+1}\right) E_{\varphi}\left(m_{i}^{*} x\right)=\sum_{i=1}^{z} m_{i} E_{\varphi}\left(m_{i}^{*} x\right)
\end{aligned}
$$

for all $x \in M$.

Now as $p_{1} \preceq p_{2} \preceq \ldots \preceq p_{n} \preceq p_{n+1}$, for $1 \leq j \leq n+1$ we can write

$$
p_{j}=\left(\sum_{i=1}^{w_{j}} q_{j, i}\right)+r_{j}, \text { with } q_{j, i} \sim p_{1}\left(=q_{1,1}\right) \text { for } 1 \leq i \leq w_{j} \text {, and } r_{j} \prec p_{1}
$$

where $\left\{q_{j, i}\right\}_{1 \leq i \leq w_{j}}$ and $r_{j}$ are orthogonal subprojections of $p_{j}$.

There exist partial isometries $\left\{v_{j, i}^{t, h}\right\}$ in $M$ with $1 \leq j, t \leq n+1,1 \leq i \leq w_{j}$ and $1 \leq h \leq w_{t}$ such that $q_{j, i} \stackrel{v_{j, i}^{t, h}}{\sim} q_{t, h}$. Therefore, they verify 


$$
\left(v_{j, i}^{t, h}\right)^{*}=v_{t, h}^{j, i}, v_{j, i}^{t, h} v_{t, h}^{j, i}=q_{t, h}, v_{j, i}^{t, h} v_{l, w}^{k, s}=\delta_{(j, i)(k, s)} v_{l, w}^{t, h}
$$

Then $v_{j, i}^{j, i}=q_{j, i}$, and $v_{j, i}^{t, h} \cdot q_{k, s} \cdot v_{t, h}^{j, i}=v_{j, i}^{t, h} v_{k, s}^{k, s} v_{t, h}^{j, i}=\delta_{(j, i)(k, s)} v_{t, h}^{t, h}=\delta_{(j, i)(k, s)} q_{t, h}$.

There also exist partial isometries $\left\{u_{j}^{t, i}\right\}$ with $1 \leq j, t \leq n+1$ and $1 \leq i \leq w_{t}$ such that $r_{j} \stackrel{u_{j}^{t, i}}{\sim} s_{t, i}<q_{t, i}$. They verify

$$
r_{j}=\left(u_{j}^{t, i}\right)^{*} u_{j}^{t, i}, \text { and } u_{j}^{t, i}\left(u_{j}^{t, i}\right)^{*}=s_{t, i}<q_{t, i}
$$

We are going to use the following equalities:

$$
\begin{gathered}
v_{j, i}^{t, h} p_{k} v_{t, h}^{j, i}=v_{j, i}^{t, h}\left(\sum_{s=1}^{w_{k}} q_{k, s}+r_{k}\right) v_{t, h}^{j, i}=\delta_{j k} q_{t, h} \\
\left(u_{j}^{t, h}\right)^{*} p_{i} u_{j}^{t, h}=\delta_{i t} r_{j}
\end{gathered}
$$

Using 4.1, for every $x \in M, 1 \leq t \leq n$ and $1 \leq j \leq w_{n+1}$ we have

$$
v_{t, 1}^{n+1, j} E_{\varphi}\left(v_{n+1, j}^{t, 1} x\right)=v_{t, 1}^{n+1, j} \sum_{i=1}^{n+1} p_{i} v_{n+1, j}^{t, 1} x p_{i}=q_{n+1, j} x p_{t} .
$$

Also, for $1 \leq t \leq n$ and $1 \leq j \leq m_{t}$,

$$
\begin{aligned}
v_{n+1,1}^{t, j} E_{\varphi}\left(v_{t, j}^{n+1,1} x\right) & =v_{n+1,1}^{t, j} \sum_{i=1}^{n+1} p_{i} v_{t, j}^{n+1,1} x p_{i} \\
& =v_{n+1,1}^{t, j} p_{n+1} v_{t, j}^{n+1,1} x p_{n+1}=q_{t, j} x p_{n+1} .
\end{aligned}
$$

For $2 \leq t \leq n+1$

$$
\begin{aligned}
\left(u_{t}^{n+1,1}\right)^{*} E_{\varphi}\left(u_{t}^{n+1,1} x\right) & =\left(u_{t}^{n+1,1}\right)^{*} \sum_{i=1}^{n+1} p_{i} u_{t}^{n+1,1} x p_{i} 4 \\
& =\left(u_{t}^{n+1,1}\right)^{*} p_{n+1} u_{t}^{n+1,1} x p_{n+1} \\
& =r_{t} x p_{n+1} .
\end{aligned}
$$

And for $1 \leq t \leq n$,

$$
\left(u_{n+1}^{t, 1}\right)^{*} E_{\varphi}\left(u_{n+1}^{t, 1} x\right)=\left(u_{n+1}^{t, 1}\right)^{*} \sum_{i=1}^{n+1} p_{i} u_{n+1}^{t, 1} x p_{i}=\left(u_{n+1}^{t, 1}\right)^{*} p_{t} u_{n+1}^{t, 1} x p_{t}=r_{n+1} x p_{t}
$$

Recall that $\sum_{t=1}^{n+1} p_{t}=1$ and $p_{t}=\left(\sum_{i=1} w_{t} q_{t, i}\right)+r_{t}$. It follows that 


$$
\begin{aligned}
x & =\left(1-p_{n+1}\right) x\left(1-p_{n+1}\right)+p_{n+1} x p_{n+1}+\left(\sum_{t=1}^{n} p_{t}\right) x p_{n+1}+p_{n+1} x\left(\sum_{t=1}^{n} p_{t}\right) \\
& =\left(1-p_{n+1}\right) x\left(1-p_{n+1}\right)+p_{n+1} x p_{n+1}+\left(\sum_{t=1}^{n}\left(\sum_{j=1}^{w_{t}} q_{t, j}\right)+r_{t}\right) x p_{n+1}+ \\
& +\left(\left(\sum_{j=1}^{w_{n+1}} q_{n+1, j}\right)+r_{n+1}\right) x\left(\sum_{t=1}^{n} p_{t}\right) \\
& =\left(1-p_{n+1}\right) x\left(1-p_{n+1}\right)+p_{n+1} x p_{n+1}+\sum_{t=1}^{n} \sum_{j=1}^{w_{t}} q_{t, j} x p_{n+1}+ \\
& +\sum_{t=2}^{n} r_{t} x p_{n+1}+\sum_{j=1}^{w_{n+1}} \sum_{t=1}^{n} q_{n+1, j} x p_{t}+\sum_{t=1}^{n} r_{n+1} x p_{t} .
\end{aligned}
$$

Then, using (1),(2),(3),(4) and (5) we obtain

$$
\begin{aligned}
x & =\sum_{i=1}^{z-1} m_{i} E_{\varphi}\left(m_{i}^{*} x\right)+\left(m_{z}+p_{n+1}\right) E_{\varphi}\left(\left(m_{z}\right)^{*} x+p_{n+1} x\right)+ \\
& +\sum_{t=1}^{n} \sum_{j=1}^{w_{t}} v_{n+1,1}^{t, j} E_{\varphi}\left(v_{t, j}^{n+1,1} x\right)+\sum_{t=2}^{n}\left(u_{t}^{n+1,1}\right)^{*} E_{\varphi}\left(u_{t}^{n+1,1} x\right)+ \\
& +\sum_{j=1}^{w_{n+1}} \sum_{t=1}^{n} v_{t, 1}^{n+1, j} E_{\varphi}\left(v_{n+1, j}^{t, 1} x\right)+\sum_{t=1}^{n}\left(u_{n+1}^{t, 1}\right)^{*} E_{\varphi}\left(u_{n+1}^{t, 1} x\right) .
\end{aligned}
$$

It is straightfoward to verify that the union of the sets $\left\{m_{i}\right\}_{1 \leq i \leq z-1}$, $\left\{m_{z}+p_{n+1}\right\},\left\{v_{n+1,1}^{t, j}\right\}_{\substack{1 \leq t \leq n \\ 1 \leq j \leq w_{t}}},\left\{v_{t, 1}^{n+1, j}\right\}_{\substack{1 \leq t \leq n \\ 1 \leq j \leq w_{n+1}}},\left\{\left(u_{t}^{n+1,1}\right)^{*}\right\}_{2 \leq t \leq n}$ and $\left\{\left(u_{n+1}^{t, 1}\right)^{*}\right\}_{1 \leq t \leq n}$ forms an orthonormal family. Therefore $E_{\varphi}$ has strongly finite index.

(ii) $\Longrightarrow$ (iii): If $\sigma(h)$ is infinite, for every $n \in \mathrm{N}$ there exist $n$ orthogonal spectral projections $\left\{p_{i}\right\}_{1 \leq i \leq n}$ of $h$ with $\sum_{1 \leq i \leq n} p_{i}=1$. Since $h$ is affiliated to $Z\left(M^{\varphi}\right)$, then $p_{i} \in Z\left(M^{\varphi}\right)$. As $M$ is a factor, we can suppose $p_{1} \preceq \ldots \preceq p_{n}$. Therefore, there exist subprojections $q_{i} \in M, q_{i} \leq p_{i}, 1 \leq i \leq n$ such that $q_{i} \sim q_{1}=p_{1}$, and $n^{2}$ partial isometries $v_{i j} \in M$ that verify:

$v_{l j} v_{l j}^{*}=q_{l}, v_{l j}^{*} v_{l j}=q_{j}, v_{i j}^{*}=v_{j i}$, and $v_{l j} v_{k r}=\delta_{j k} v_{l r}$ with $1 \leq l, j, k, r \leq n$.

Observe that $p_{k} v_{i j} p_{k}=\delta_{i k} \delta_{j k} v_{i j}$.

Let $v=\sum_{l, j=1}^{n} v_{l j}$. Then

$$
v^{2}=\sum_{l, j, k, r=1}^{n} v_{l j} v_{k r}=\sum_{l, j, r=1}^{n} v_{l r}=n \sum_{l, r=1}^{n} v_{l r}=n v
$$

Then $v \geq 0$ and $\|v\|=n$. 
Using lemma 4.1, $E_{\varphi}(v)=E_{\varphi}\left(\sum_{i \in I} p_{i} v p_{i}\right)$, and then

$$
E_{\varphi}(v)=E_{\varphi}\left(\sum_{1 \leq i, j, k \leq n} p_{k} v_{i j} p_{k}\right)=E_{\varphi}\left(\sum_{k=1}^{n} v_{k k}\right)=\sum_{k=1}^{n} E_{\varphi}\left(q_{k}\right)
$$

But $p_{i} E_{\varphi}\left(q_{k}\right) p_{i}=E_{\varphi}\left(p_{i} q_{k} p_{i}\right)=\delta_{i k} E_{\varphi}\left(q_{k}\right)$, and then $E_{\varphi}\left(q_{k}\right) \in p_{k} M p_{k}$. Therefore

$$
\left\|E_{\varphi}(v)\right\|=\left\|\sum_{k} E_{\varphi}\left(q_{k}\right)\right\| \geq\left\|E_{\varphi}\left(q_{1}\right)\right\|=\left\|E_{\varphi}\left(p_{1}\right)\right\|=\left\|p_{1}\right\|=1
$$

because $q_{1}=p_{1} \in M^{\varphi}$. In other words, for every $n \in \mathrm{N}$ there exists a positive element $v \in M$ such that $\left\|E_{\varphi}(v)\right\| \geq \frac{1}{n}\|v\|$. This proves that $E_{\varphi}$ has infinite weak index.

REMARK 4.3. With the same hypothesis of 4.2 , but for $M$ a factor, it is straightforward to verify that if $\sigma(h)$ consists of $n$ points, then

$$
\operatorname{Ind}\left(E_{\varphi}\right)=n .
$$

If $M$ has finite dimensional center there exists $\left\{q_{i}\right\}_{1 \leq i \leq w} \subset Z(M)$ such that $E_{\varphi}=\left.\sum_{i=1}^{w} E_{\varphi}\right|_{M q_{i}}$, where $M q_{i}$ is a finite factor. In that case, $\operatorname{Ind}\left(\left.E_{\varphi}\right|_{M q_{i}}\right)=$ $n_{i}$, where $n_{i}$ is the number of points of $\sigma\left(h_{i}\right)\left(h_{i}=\left.h\right|_{M q_{i}} \in L\left(M q_{i}\right)\right)$. Then the strong index

$$
\operatorname{Ind}\left(E_{\varphi}\right)=\sum_{i=1}^{w} n_{i} q_{i} \in Z(M)
$$

The analogous result for the semifinite case also holds.

THEOREM 4.4. Let $M$ be a semifinite von Neumann algebra with finite dimensional center, $\varphi$ a faithful normal strictly semifinite weight and $E_{\varphi}: M \longrightarrow M^{\varphi}$ the unique normal and faithful conditional expectation that leaves $\varphi$ invariant. Let $\tau$ be a tracial weight in $M$ and $h$ affiliated to $Z\left(M^{\varphi}\right)$ such that $\varphi=\tau(h$.). The following statements are equivalent:

(i) $E_{\varphi}$ has weakly finite index.

(ii) $\sigma(h)$ is finite.

Proof. (ii) $\Longrightarrow$ (i): If $\sigma(h)$ is finite, denote by $p_{i}$ the spectral projections of $h$. It is a standard fact that the expectation $E_{\varphi}(x)=\sum_{i=1}^{n} p_{i} x p_{i}$ has weakly finite index.

(i) $\Longrightarrow$ (ii) follows exactly as in the previous theorem.

REMARK 4.5. If $\operatorname{dim}(Z(M))$ is not finite, the preceeding results are not true. Take for example the finite von Neumann algebra $M=\oplus_{n \in \mathrm{N}} M_{2}(\mathrm{C})$ 
(where $M_{2}(\mathrm{C})$ are the $2 \times 2$ matrices). Put $h=\oplus_{n} h_{n}$ with $h_{n} \in M_{2}(\mathrm{C})$ the diagonal matrix $h_{n}=\left(\begin{array}{cc}1 / n & 0 \\ 0 & 1+1 / n\end{array}\right)$. Let $\tau$ be the trace on $M$ defined by $\tau\left(\oplus_{n} x_{n}\right)=\sum_{n} \frac{1}{2^{n}} \operatorname{tr}\left(x_{n}\right)$, where $\operatorname{tr}$ is the canonical trace on $M_{2}(\mathrm{C})$. Then, $\varphi()=.\tau\left(\frac{h}{\tau(h)}\right.$. $)$ defines a faithful normal state with $M^{\varphi}=M \cap\{h\}^{\prime}=$ $\left\{\oplus_{n} x_{n}: x_{n}\right.$ is a diagonal matrix $\}$. Then if $D\left(\begin{array}{cc}z_{11} & z_{12} \\ z_{21} & z_{22}\end{array}\right)=\left(\begin{array}{cc}z_{11} & 0 \\ 0 & z_{22}\end{array}\right)$, it is clear that $E_{\varphi}\left(\oplus_{n} z_{n}\right)=\oplus_{n} D\left(z_{n}\right)$.

$E_{\varphi}$ has strongly finite index: put $u_{1}=1 \in M, u_{2}=\oplus_{n}\left(\begin{array}{cc}0 & 1 \\ 0 & 0\end{array}\right)$ and $u_{3}=\oplus_{n}\left(\begin{array}{ll}0 & 0 \\ 1 & 0\end{array}\right)$, it is easy to see that for every $x \in M, x=\sum_{i=1}^{3} u_{i} E_{\varphi}\left(u_{i}^{*} x\right)$ and that $E_{\varphi}\left(u_{i}^{*} u_{j}\right)=0$ if $i \neq j$.

Observe that if $\varphi()=.\mu\left(k\right.$.) with $\mu$ a trace on $M$ and $k=\oplus_{n} k_{n} \in M_{+}^{\varphi}$. Then the spectrum of $k$ has to be infinite. Indeed, $\mu$ is a multiple of $\operatorname{tr}$ on each $M_{2}$ (C) (let us say $\mu\left(z_{n}\right)=\alpha_{n} \operatorname{tr}\left(z_{n}\right)$ ) and therefore $\frac{1}{2^{n}} \operatorname{tr}\left(h_{n}\right.$.) $=\alpha_{n} \operatorname{tr}\left(k_{n}\right.$.) for every $n \in \mathrm{N}$. Clearly this equality would not hold if the spectrum of $k$ is finite.

Summarizing, we have found a faithful normal state $\varphi$ on $M$, with $E_{\varphi}$ of strongly finite index, such that for every trace $\mu$ on $M$, if $\varphi()=.\mu(k$.) then the spectrum of $k$ is infinite.

It has been already observed (3.2) that if $M$ is a type III algebra, there are no normal conditional expectations of finite index onto $M^{\varphi}$. Moreover, if $M$ is a factor of type III there cannot exist $E_{\varphi}$ with weakly finite index.

PROPOSITION 4.6. Let $M$ be a factor of type III, and $\varphi$ a faithful normal and strictly semifinite weight in $M$. Then the weak index of $E_{\varphi}$ is infinite.

Proof. By the result of [AS] cited above, if $E_{\varphi}$ had weakly finite index, then $M^{\varphi}$ should have finite dimensional center. Let $p_{1}, \ldots, p_{n}$ be the minimal projections of the center of $M^{\varphi}$. Then the expectations

$$
E_{i}: M_{p_{i}} \rightarrow p_{i} M^{\varphi} \quad E_{i}\left(p_{i} x p_{i}\right)=E_{\varphi}\left(p_{i} x p_{i}\right)
$$

have weak finite index, for $i=1, \ldots n$. But since $p_{i} M p_{i}$ and $p_{i} M^{\varphi}$ are factors, the expectations $E_{i}$ have strongly finite index. Therefore $\sum_{i} E_{i}$ : $\oplus p_{i} M p_{i} \rightarrow M^{\varphi}$ has strongly finite index. Note that $E=\left(\sum_{i} E_{i}\right) \circ F$, where $F(x)=\sum_{i} p_{i} x p_{i}$. The projections $p_{1}, \ldots, p_{n}$ are equivalent in $M$. Therefore an 
argument analogous (but much simpler) to that of ii) $\Rightarrow$ i) in 4.2 , shows that $F$ has strongly finite index. Therefore $E_{\varphi}$ has strongly finite index, which leads to a contradiction.

\section{The geometry of the orbit of a faithful, normal, strictly semifinite weight}

Let us introduce a case where the unitary orbits of faithful and normal states are not (topological) submanifolds of the predual (and neither the dual) space of the algebra. In the case of orbits of weights, no natural ambient space is given in order to model the manifold structure of the orbit. This facts justify the use of other topologies other than the usual norm topology.

Proposition 5.1. Let $\varphi$ be a faithful, normal state of $\mathscr{L}(H)$ with $H$ infinite dimensional. Then the unitary orbit $\mathscr{U}_{\varphi}=\{\varphi \circ \operatorname{Ad}(u): u \in \mathscr{U}(\mathscr{L}(H))\}$ is not a topological submanifold of $\mathscr{L}(H)_{*}$ (and neither of $\left.\mathscr{L}(H)^{*}\right)$.

Proof. There exists $a \in \mathscr{L}(H)_{+}$with $\operatorname{Tr}(a)=1$ ( $\operatorname{Tr}$ the usual trace), such that $\varphi(x)=\operatorname{Tr}(a x)$ for all $x \in \mathscr{L}(H)$. The element $a$ is of the form $a=\sum_{i=1}^{\infty} \lambda_{i} p_{i}$ with $\operatorname{dim} R\left(p_{i}\right)=m_{i}<\infty$, and therefore $\sum_{i=1}^{\infty} \lambda_{i} m_{i}=1$. Moreover, the unitary orbit $\mathscr{U}_{\varphi} \subset \mathscr{L}(H)_{*}$ with the norm topology identifies with $\mathscr{U}_{a}=\left\{u^{*} a u: u \in \mathscr{U}(\mathscr{L}(H))\right\} \subset \mathscr{T}(H)$ with the trace norm \|\|$_{1}$, where $\mathscr{T}(H)$ denotes the trace class of $\mathscr{L}(H)$.

Let $u_{n}$ be unitaries in $\mathscr{L}(H)$ such that $u_{n}^{*} a u_{n}$ converge to $b$ in the usual norm of $\mathscr{L}(H)$.

Then it can be proved that

i) $b$ is compact and positive,

ii) $\sigma(b)=\left\{\lambda_{i}: i \in \mathbf{N}\right\}=\sigma(a), \quad b=\sum_{i=1}^{\infty} \lambda_{i} q_{i}$ with $\operatorname{dim} R\left(q_{i}\right)=m_{i}$, and $\operatorname{Tr}(b)=1$,

iii) $u_{n}^{*} a u_{n} \rightarrow b$ in \|\|$_{1}$.

If $\mathscr{U}_{a}=\left\{u^{*} a u: u \in \mathscr{U}(\mathscr{L}(H))\right\}$ is a submanifold of $\left(\mathscr{T}(H),\|\|_{1}\right)=\mathscr{L}(H)_{*}$ then $\mathscr{U}_{a}$ is locally closed in $\mathscr{T}(H)$. That is, each point $c \in \mathscr{U}_{a}$ has a neighbourhood of the form $\left\{d \in \mathscr{U}_{a}:\|c-d\|_{1} \leq \varepsilon\right\}$ which is closed in $\mathscr{T}(H)$. But since the action of the unitaries of $\mathscr{L}(H)$ is isometric on $\mathscr{T}(H)$, the number $\varepsilon$ can be chosen the same for all $c$ in $\mathscr{U}_{a}$. This clearly implies that the orbit $\mathscr{U}_{a}$ is closed in $\mathscr{T}(H)$. Therefore, by the remarks above, $\mathscr{U}_{a}$ is closed in the usual norm of $\mathscr{L}(H)$. In his remarkable paper [V], Voiculescu proved, as a byproduct of his non-commutative Weyl-von Neumann theorem, that this condition - closedness in norm of the unitary orbit of an operator in $\mathscr{L}(H)$ implies that the operator generates a finite dimensional $\mathrm{C}^{*}$-algebra. In our case, since $a$ is positive, this implies that the spectrum of $a$ is finite. This leads to a contradiction, since $a$ is also compact and has zero kernel.

Let $\varphi$ be a faithful, normal, strictly semifinite weight on a von Neumann 
algebra $M$. Let $\left(H_{\varphi}, \pi_{\varphi}, \eta_{\varphi}\right)$ be the GNS triple as in 1.2 , and $E: M \rightarrow N$ a $\varphi$ invariant conditional expectation onto a von Neumann subalgebra $N \subset M$. Let $e \in L\left(H_{\varphi}\right)$ be the Jones projection associated to $E$, that is $e$ is the orthogonal projection on $\overline{N \cap \mathcal{N}_{\varphi}} \subset H_{\varphi}$, and $M_{1}=(M, e)^{\prime \prime} \subset L\left(H_{\varphi}\right)$ the basic extension. From now on we shall identify $x \in M$ with its image $\pi_{\varphi}(x) \in \mathscr{L}\left(H_{\varphi}\right)$.

The orbit $\mathscr{U}_{\varphi}=\{\varphi \circ \operatorname{Ad}(u): u \in \mathscr{U}(M)\}$ is not "included" in any Banach space. Nevertheless, we shall introduce a representation for $\mathscr{U}_{\varphi}$ which will allow us to present it as a space of projections of the basic extension of $M$ by $E_{\varphi} \cdot \mathscr{U}_{\varphi}$ will be considered with the quotient topology $\mathscr{U}(M) / \mathscr{U}\left(M^{\varphi}\right)$.

If $\varphi$ is a faithful, normal, strictly semifinite weight, let $e_{\varphi}$ be the Jones projection of $E_{\varphi}: M \rightarrow M^{\varphi}$. The orbit $\mathscr{U}_{M}\left(e_{\varphi}\right)=\left\{u^{*} e_{\varphi} u: u \in \mathscr{U}(M)\right\}$ is a $C^{\infty}$ homogeneous space [AV]. The continuous map (called the basic representation of $\mathscr{U}_{\varphi}$ )

$$
\begin{gathered}
\beta: \mathscr{U}_{\varphi} \rightarrow \mathscr{U}_{M}\left(e_{\varphi}\right) \subset M_{1} \\
\beta(\varphi \circ \operatorname{Ad}(u))=u^{*} e_{\varphi} u
\end{gathered}
$$

is a bijection that preserves the adjoint and the orbits.

The tangent space $T\left(\mathscr{U}_{M}\left(e_{\varphi}\right)\right)_{e_{\varphi}}$ identifies with the space $\left\{x e_{\varphi}-e_{\varphi} x\left(=\left[x, e_{\varphi}\right]\right): x \in M, x^{*}=-x\right\}$.

$\mathscr{U}_{M}\left(e_{\varphi}\right) \subset M_{1}$ is a Banach homogeneous space, consisting of projections of $M_{1}$. It has a natural connection (see [ALRS]), for example, its geodesics can be easily computed. Namely, the unique geodesic $\varphi_{t}$ on $\mathscr{U}_{M}\left(e_{\varphi}\right)$ with $\varphi_{0}=e_{\varphi}$ and $\left.\frac{d}{d t} \varphi_{t}\right|_{t=0}=\left[x, e_{\varphi}\right]$ is given by

$$
\varphi_{t}=e^{t x_{0}} e_{\varphi} e^{-t x_{0}}
$$

where $x_{0}=x-E_{\varphi}(x)$.

REMARK 5.2. a) One has the following commutative diagram

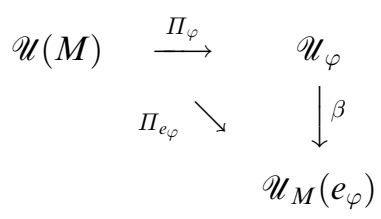

where $\Pi_{\varphi}(u)=\varphi \circ \operatorname{Ad}(u)$ and $\Pi_{e_{\varphi}}(u)=u^{*} e_{\varphi} u$, for $u \in \mathscr{U}(M)$. The map $\Pi_{e_{\varphi}}$ has local cross sections, namely (near $e_{\varphi}$ ):

$$
s\left(u^{*} e_{\varphi} u\right)=\left(E_{\varphi}(u) E_{\varphi}\left(u^{*}\right)\right)^{-1 / 2} E_{\varphi}(u) u,
$$

(i.e. the unitary part of $E_{\varphi}(u) u$ in its polar decomposition) defined in $\left\{u^{*} e_{\varphi} u:\left\|u^{*} e_{\varphi} u-e_{\varphi}\right\|<1\right\}$, which takes values in $\mathscr{U}(M)$ and satisfies 


$$
\Pi_{e_{\varphi}} \circ s\left(u^{*} e_{\varphi} u\right)=u^{*} e_{\varphi} u \text { and } s\left(e_{\varphi}\right)=1 .
$$

Note that if $\left\|u^{*} e_{\varphi} u-e_{\varphi}\right\|<1$ then also $\left\|e_{\varphi} u^{*} e_{\varphi} u e_{\varphi}-e_{\varphi}\right\|$ and $\| e_{\varphi}-$ $e_{\varphi} u e_{\varphi} u^{*} e_{\varphi} \|$ are strictly less than 1 , which implies (using the properties of the basic extension) that $\left\|E_{\varphi}\left(u^{*}\right) E_{\varphi}(u)-1\right\|<1$ and $\left\|1-E_{\varphi}(u) E_{\varphi}\left(u^{*}\right)\right\|<1$, and therefore $s$ is well defined.

b) If $M$ is endowed with the left $M^{\varphi}$-Hilbert module norm induced by $E_{\varphi}$, i.e. $\|x\|_{E_{\varphi}}=\left\|E_{\varphi}\left(x^{*} x\right)\right\|^{1 / 2}$, then $s$ is continuous. This is a straightforward verification, using the fact noted in a), that if $u^{*} e_{\varphi} u$ is close to $e_{\varphi}$ then $E_{\varphi}\left(u^{*}\right) E_{\varphi}(u)$ and $E_{\varphi}(u) E_{\varphi}\left(u^{*}\right)$ are close to 1 . Now, if $\Pi_{e_{\varphi}}$ has continuous local cross section, by means of the diagram above it is easy to see that the basic representation $\beta$ is a homeomorphism. Therefore $\mathscr{U}_{\varphi}$ with the quotient topology $\left(\mathscr{U}(M),\|\|_{E_{\varphi}}\right) / \mathscr{U}\left(M^{\varphi}\right)$ can be regarded as a manifold of projections of $M_{1}$.

c) It is known that the equivalence in $M$ of the usual norm with the Hilbert module norm is equivalent to the finite index condition. Therefore if the index of $E_{\varphi}$ is finite, then $\mathscr{U}_{\varphi}$ is homeomorphic to $\mathscr{U}_{M}\left(e_{\varphi}\right)$, with the usual norms.

We do not know if $\beta$ is (norm) continuous in general. In other words, if the Hilbert module norm and the usual norm of $M$ can induce the same quotient topology in $\mathscr{U}(M) / \mathscr{U}\left(M^{\varphi}\right)$ in cases other than the finite index situation.

If $\operatorname{Ind}\left(E_{\varphi}\right)<\infty$ one can do more. Let us recall the following result from [AS2].

TheOrem 5.3. Let $N \subset M$ be a von Neumann algebra, $E: M \rightarrow N$ a normal and faithful conditional expectation and $e$ and $M_{1}$ as before. Then, the following statements are equivalent

1) The weak index of $E$ is finite.

2) $\mathscr{S}_{M}(e)=\left\{\mathrm{geg}^{-1}: g\right.$ invertible in $\left.M\right\}$ is an analytic homogeneous $\mathrm{Ba}$ nach space under the action of the invertible elements of $M$ and an analytic submanifold of $M_{1}$.

3) $\mathscr{U}_{M}(e)=\left\{\right.$ ueu $\left.^{*}: u \in \mathscr{U}(M)\right\}$ is a $C^{\infty}$ homogeneous Banach space under the action of the unitary elements of $M$ and a $C^{\infty}$ submanifold of $M_{1}$.

Corollary 5.4. Let $M$ be a von Neumann algebra with finite dimensional center and $\varphi$ a faithful, normal and strictly semifinite weight. The following statements are equivalent:

1) $E_{\varphi}$ has weakly finite index.

2) $M$ is semifinite and $\sigma(h)$ is finite, if $h$ is the Radon-Nikodym derivative of the weight $\varphi$ with respect to a faithful, normal and semifinite trace on $M$. 
3) $\mathscr{U}_{M}\left(e_{\varphi}\right)=\left\{u e_{\varphi} u^{*}: u \in \mathscr{U}(M)\right\}$ is a $C^{\infty}$ Banach homogeneous space under the action of the unitaries of $M$ and a $C^{\infty}$ submanifold of $M_{1}=(M, e)^{\prime \prime}$.

If the weak index of $E_{\varphi}$ is infinite, $\mathscr{U}_{M}\left(e_{\varphi}\right)$ and $\mathscr{S}_{M}\left(e_{\varphi}\right)$ are manifolds of projections of $M_{1}$, but not submanifolds of $M_{1}$.

Proof. 1) $\Rightarrow 2$ 2) $M$ is semifinite by 3.2 and by theorem $4.4 \sigma(h)$ is finite.

2) $\Rightarrow 1$ ) by 4.4 .

1) $\Leftrightarrow 3$ ) by 5.3 .

REMARK 5.5. As we noted before, these conditions allow us to give a differential structure to $\mathscr{U}_{\varphi}$ the orbit of a faithful, normal and strictly semifinite weight $\varphi$, through the model

$$
\begin{gathered}
\varphi \circ \operatorname{Ad}(u) \mapsto u^{*} e_{\varphi} u \\
\mathscr{U}_{\varphi} \leftrightarrow \mathscr{U}_{M}\left(e_{\varphi}\right) .
\end{gathered}
$$

Note that if $E_{\varphi}$ has finite index, then with the notations above, $M^{\varphi}=\{h\}^{\prime} \cap M$. Therefore, $\left(M^{\varphi}\right)^{\prime} \cap M \subset M^{\varphi}$. Indeed, since if $p_{1}, \ldots, p_{n}$ are the minimal spectral projections of $h$, then $\left(M^{\varphi}\right)^{\prime}$ consists of the elements of $M$ wich have "diagonal" matrices with respect to $p_{1}, \ldots, p_{n}$.

Recall again from [AS2], that if $E: M \rightarrow N$ has finite index and $N^{\prime} \cap M \subset N$, then the mapping

$$
\mathscr{U}_{M}(e)=\left\{u^{*} e u: u \in \mathscr{U}(M)\right\} \longrightarrow \mathcal{O}_{E}=\left\{\operatorname{Ad}(u) \circ E \circ \operatorname{Ad}\left(u^{*}\right): u \in \mathscr{U}(M)\right\}
$$

is a covering map with fibre homeomorphic to $n(E) / \mathscr{U}(N)$, where $n(E)=\{u \in \mathscr{U}(M): \operatorname{Ad}(u)(N) \subset N\}$.

In our case, we obtain that if $\varphi$ is a weight on $M$ satisfying the equivalent conditions of 5.4 , then the map

$$
\mathscr{U}_{\varphi} \longrightarrow \mathcal{O}_{E_{\varphi}}=\left\{\operatorname{Ad}(u) \circ E_{\varphi} \circ \operatorname{Ad}\left(u^{*}\right): u \in \mathscr{U}(M)\right\}
$$

is a covering map with fibre (homeomorphic to) the group $n\left(E_{\varphi}\right) / \mathscr{U}\left(M^{\varphi}\right)$. Moreover, since $Z(M)$ is finite dimensional, it can be shown that this group is finite (see $[\mathrm{ArS}])$.

\section{REFERENCES}

[AS] E. Andruchow and D. Stojanoff, Group conditional expectations of weak finite index, Bol. Acad. Nac. Cs. (Córdoba), to appear (1998)..

[AS2] E. Andruchow and D. Stojanoff, Geometry of conditional expectations and finite index, International J. Math. 5 (1994), 169-178.

[AV] E. Andruchow and A. Varela, Geometry and the Jones projection of a state, Integral Equations Operator Theory 25 (1996), 129-146. 
[ArS] M. Argerami and D. Stojanoff, A discrete group associated to a finite index conditional expectation, Integral Equations Operator Theory, to appear.

[BDH] M. Baillet, Y. Denizeau and J.-F. Havet, Indice d'une esperance conditionelle, Compositio Math. 66 (1988), 199-236.

[C] F. Combes, Poids associes a une algebre hilbertienne a gauche, Compositio Math. 23, fasc. 1 (1971), 4-77.

[Co] A. Connes, Une classification des facteurs de type III, Ann. Sci. Ecole Norm. Sup., 6 (1973), 133-252.

[CPR] G. Corach, H. Porta and L. Recht, The geometry of spaces of projections in C*-algebras, Adv. Math. 101 (1993), 59-77.

[D] J. Dixmier, Von Neumann Algebras, North-Holland, Amsterdam, 1981.

[E] W. Eberlein, Abstract ergodic theorems and weak almost periodic functions, Trans. Amer. Math. Soc. 67 (1949), 217-240.

[F] M. Frank, Normal operator-valued weights between monotone complete $C^{*}$ algebras, preprint (1995).

[FK] M. Frank and E. Kirchberg, On conditional expectations of finite index, J. Operator Theory 10 (1998), 87-111.

[H] U. Haagerup, The standard form of a von Neumann algebra, Math. Scand. 37 (1975), 271-283.

[Ha] J.-F. Havet, Esperance conditionelle minimal, J. Operator Theory 24 (1990), 33-55.

[J] P. Jolissaint, Indice d'esperances conditionelles et algebres de von Neumann finies, Math. Scand. 68 (1991), 221-246.

[Jo] V.F.R. Jones, Index for subfactors, Invent. Math. 72 (1983), 1-25.

[KR] R.V. Kadison and J.R. Ringrose, Fundamentals of the Theory of Operator Algebras I, II, Academic Press, New York 1984, 1986.

[K] H. Kosaki, Extension of Jones' index theory to arbitrary factors, J. Funct. Anal. 66 (1986), 123-140.

[Pa] A.T. Paterson, Amenability, Math. Surveys Monographs, 1988.

[P] G.K. Pedersen, $C^{*}$-Algebras and their automorphism groups, Academic Press, New York, 1979.

[PT] G.K. Pedersen and M. Takesaki, The Radon-Nikodym theorem for von Neumann algebras, Acta Math. 130 (1973), 53-87.

[PP] M. Pimsner and S. Popa, Entropy and index for subfactors, Ann. Sci. Ecole Norm. Sup. 19 (1986), 57-106.

[W] Y. Watatani, Index for $C^{*}$-subalgebras, Mem. Amer. Math. Soc. 83 (1990).

[Su] F.D. Suárez, Conditional expectations in $L^{2}(0,1)$, preprint.

[S] S. Stratila, Modular theory in operator algebras, Abacus Press, Kent, 1981.

[T] M. Takesaki, Conditional expectations in von Neumann algebras, J. Funct. Anal. 9 (1972), 306-321.

[U] H. Umegaki, Conditional expectation in operator algebra III, Kodai Math. Sem. Rep. 11 (1959), 51-64.

[V] D.V. Voiculescu, A non commutative Weyl-von Neumann theorem, Rev. Roum. Math. Pures Appl. 21 (1976), 97-113.

INSTITUTO DE CIENCIAS

UNIVERSIDAD NACIONAL DE GRAL. SARMIENTO

J. A. ROCA 850

(1663) SAN MIGUEL

ARGENTINA

e-mail: eandruch@mate.dm.uba.ar, ale@alevar.uba.ar 